\title{
口m
}

BioMedical Engineering

OnLine

The baseline pressure of intracranial pressure (ICP) sensors can be altered by electrostatic discharges

Eide and Bakken 


\section{The baseline pressure of intracranial pressure (ICP) sensors can be altered by electrostatic discharges}

Per K Eide ${ }^{1,2^{*}}$ and André Bakken ${ }^{3}$

\author{
* Correspondence: per.kristian. \\ eide@ous-hf.no \\ ${ }^{1}$ Department of Neurosurgery, Oslo \\ University Hospital, Rikshospitalet, \\ Oslo, Norway \\ Full list of author information is \\ available at the end of the article
}

\begin{abstract}
Background: The monitoring of intracranial pressure (ICP) has a crucial role in the surveillance of patients with brain injury. During long-term monitoring of ICP, we have seen spontaneous shifts in baseline pressure (ICP sensor zero point), which are of technical and not physiological origin. The aim of the present study was to explore whether or not baseline pressures of ICP sensors can be affected by electrostatics discharges (ESD's), when ESD's are delivered at clinically relevant magnitudes.

Methods: We performed bench-testing of a set of commercial ICP sensors. In our experimental setup, the ICP sensor was placed in a container with $0.9 \% \mathrm{NaCl}$ solution. A test person was charged $0.5-10 \mathrm{kV}$, and then delivered ESD's to the sensor by touching a metal rod that was located in the container. The continuous pressure signals were recorded continuously before/after the ESD's, and the pressure readings were stored digitally using a computerized system

Results: A total of 57 sensors were tested, including 25 Codman ICP sensors and 32 Raumedic sensors. When charging the test person in the range $0.5-10 \mathrm{kV}$, typically ESD's in the range $0.5-5 \mathrm{kV}$ peak pulse were delivered to the ICP sensor. Alterations in baseline pressure $\geq 2 \mathrm{mmHg}$ was seen in 24 of 25 (96\%) Codman sensors and in 17 of 32 (53\%) Raumedic sensors. Lasting changes in baseline pressure $>10 \mathrm{mmHg}$ that in the clinical setting would affect patient management, were seen frequently for both sensor types. The changes in baseline pressure were either characterized by sudden shifts or gradual drifts in baseline pressure.

Conclusions: The baseline pressures of commercial solid ICP sensors can be altered by ESD's at discharge magnitudes that are clinically relevant. Shifts in baseline pressure change the ICP levels visualised to the physician on the monitor screen, and thereby reveal wrong ICP values, which likely represent a severe risk to the patient.
\end{abstract}

\section{Background}

In patients with brain injury due to traumatic brain injury, stroke, or complications to neurosurgery, the continuous monitoring of intracranial pressure (ICP) is crucial for surveillance [1-3], even though no randomized trials have confirmed the benefit of ICP monitoring in patients with brain injury [4].

Modern ICP monitoring was first introduced by Janny in 1950 [5] and Lundberg in 1960 [6]. While ICP initially was mostly measured from fluid-filled catheters in 
connection with the ventricular cerebrospinal fluid (CSF), the first ICP micro transducers were introduced in the 1980's [7,8]. The ICP micro transducers most commonly used today include Camino [9] and Codman [10] ICP sensors which were introduced in the 1980's, the Spiegelberg ICP sensor [11] introduced in the 1990's, the Raumedic ICP sensor introduced in the beginning of 2000 [12], and the Pressio ICP sensor [13] introduced more recently. There is an extensive literature on the assessment of these ICP sensors, including bench testing [10,11,13-19] and clinical evaluation [9,12,20-30].

In our hospital, we have particularly addressed the problem of spontaneous shifts in baseline pressure (zero point) that occur during continuous ICP monitoring. Simultaneous monitoring from two ICP sensors placed nearby in the brain demonstrated spontaneous shifts in baseline pressure, which produced differences in ICP even $>20$ $\mathrm{mmHg}$ [31]. Since the differences in ICP were accompanied by close to identical ICP waveforms, the differences in ICP could be explained by shifts in baseline pressure of technical, not physiological, origin. Similar observations of marked differences in ICP even $>10-20 \mathrm{mmHg}$ despite identical ICP waveform were done when the ICP sensors were placed in different intracranial locations [30,32]. Moreover, during long-term ICP monitoring, sudden shifts in baseline ICP occurred with few hours interval [33]. The reasons for spontaneous shifts in baseline pressure have not been identified.

The present study was undertaken to explore whether or not commercial ICP sensors are affected by electrostatic discharges (ESD's). To our knowledge this topic has previously not been addressed. The issue of electrical safety in hospitals has received much attention since many years [34-37]. The need for increased awareness of electromagnetic interference with medical equipment also was addressed more recently [38]. In the hospital environment, ESD's can be evoked during patient care such as bedding of hospital beds [39]. The frequency and severity of ESD's are affected by numerous factors such as humidity and temperature, the in-house environment (textiles used in clothing, antistatic floor and washing).

In this study, we made an experimental set-up to deliver ESD's to ICP sensors at magnitudes that are clinically relevant. We tested different types of solid ICP sensors that have previously been extensively tested and are still widely used, namely the Codman ICP sensor, and the Raumedic NeuroVent and NeuroDur ICP sensors.

\section{Methods}

ICP sensors

The following types of commercially available ICP sensors were tested: Codman ICP MicroSensor (Codman, Johnson \& Johnson, Raynham, MA, USA; Figure 1a), Raumedic NeuroVent P-C (Raumedic AG, Münchberg, GE; Figure 1b), Raumedic NeuroVent P (Raumedic AG, Münchberg, GE; Figure 1c), and Raumedic NeuroDur sensor (Raumedic AG, Münchberg, GE; Figure 1d).

Those ICP sensors that had previously been used in patients were stored in closed plastic bags at room temperature.

This study did not include research on humans or animals; ethical approval for the study was not applicable. 


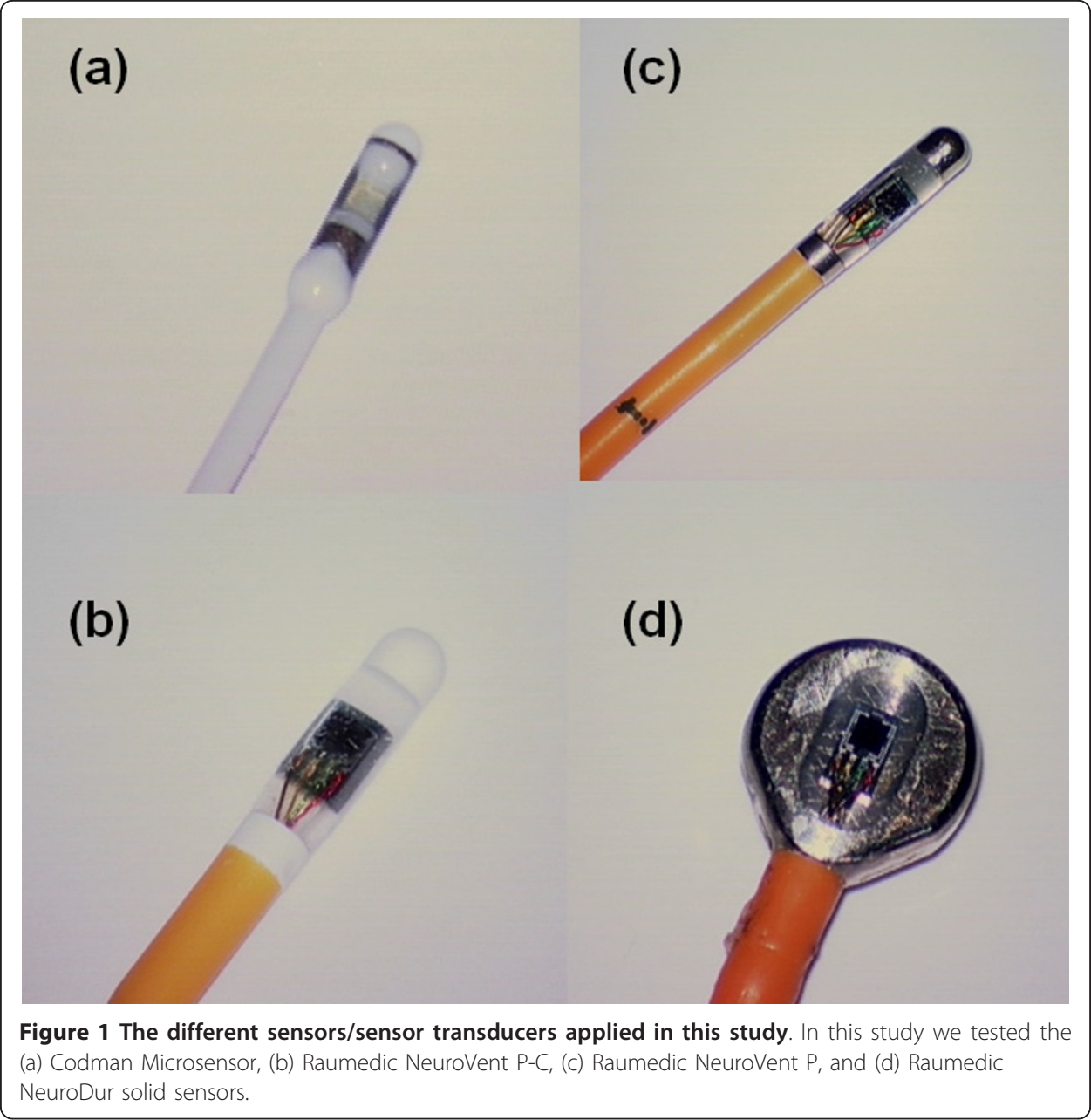

\section{Experimental setup}

The experimental setup is illustrated in Figure 2. The sensor was placed in a container filled with $0.9 \% \mathrm{NaCl}$ solution. Also a metal rod was placed in the container, whereby a test-person could deliver ESD's to the sensor. The sensor cable was wrapped around the container in order to compare with the clinical situation, wherein the cable is placed on the patient, resulting in increased capacitance. The wrapping procedure was not done for SensorID's 1-3. The Codman sensor was connected to a Codman ICP Express (Codman, Johnson \& Johnson, Raynham, MA, USA), which is a pressure transducer. The Raumedic sensors were connected to a MPR1 Raumedic pressure transducer (Raumedic AG, Münchberg, GE). The continuous ICP signals from the Codman ICP express were sampled at $200 \mathrm{~Hz}$ using the Sensometrics pressure logger, which is an analogue to digital converter, and stored on a computer using the Sensometrics ${ }^{\circledR}$ software (dPCom A/S, Oslo). The continuous signals provided by the Raumedic MPR1 were transferred directly to the computer and stored using Sensometrics ${ }^{\circledR}$ software.

In order to record the electrostatic levels reached, we used the Stat Arc II model 265 (Monroe Electronics Inc., 100 Housel Ave., Lyndonville, N.Y.).

The testing was done in standardized room temperature of $22-23^{\circ} \mathrm{C}$. 


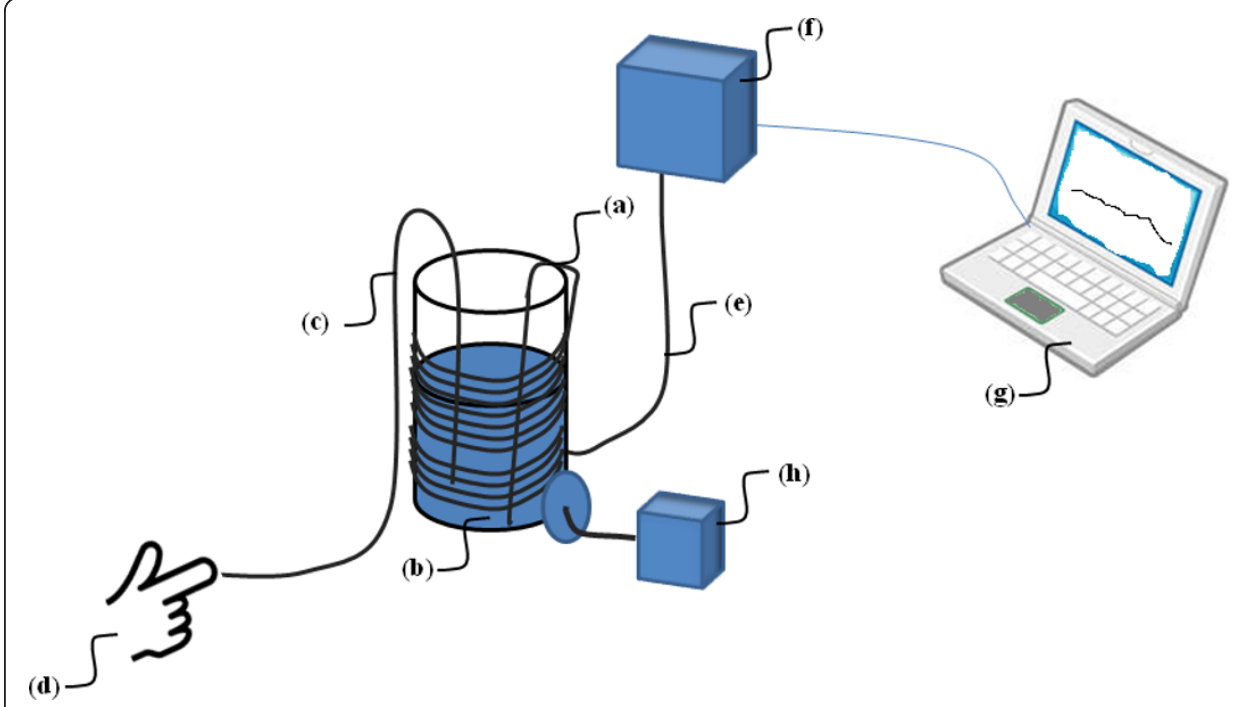

Figure 2 Experimental set-up. The ICP sensor (a) was placed in a container (b) containing $0.9 \% \mathrm{NaCl}$ solution. A metal rod (c) was placed in the $0.9 \% \mathrm{NaCl}$ solution; thereby the test person (d) could deliver ESD to the sensor (a). The ICP sensor was connected via a cable (e) to the pressure transducer (f), which was the Codman ICP Express for Codman sensors, and Raumedic MPR-1 for the various Raumedic sensors. The pressure transducer ( $f$ ) was further connected with a laptop computer ( $g$ ) with Sensometrics software for sampling and storage of the continuous pressure signals. In order to record the ESD delivered to the sensor, a meter (h) was connected with the container to read the magnitude of ESD.

\section{Testing of effects of electrostatic discharges}

After initiating the pressure recording, the test person was charged using a Metriso 5000 insulation tester (Metrawatt $\mathrm{GmbH}$, Germany) and thereafter touching the metal rod. The test-person was charged in the following sequence: $0.5 \mathrm{kV}, 1.0 \mathrm{kV}, 2.5 \mathrm{kV}$, and $5.0 \mathrm{kV}$. For deliverance of $10 \mathrm{kV}$, the test person first charged the container to $5 \mathrm{kV}$ and then swiftly charged himself to $5 \mathrm{kV}$ opposite polarity before touching the metal rod. The test-person noted whether or not he detected the current impulse being delivered.

The testing of a Codman sensor is shown in Additional file 1, and the testing of a Raumedic sensor is shown in Additional file 2.

\section{Assessment of leakage current}

All sensors were tested for leakage current using the Metriso 5000 insulation tester and a Fluke 87 III True RMS Multimeter (in the voltage range). The procedure of testing for leakage current is shown in Figure 3. All leakage current measurements were done at $500 \mathrm{~V}$ unless otherwise stated; resistance was calculated.

\section{Results}

\section{ICP sensors}

We tested a total of 57 sensors (25 Codman and 32 Raumedic sensors). Six Codman sensors (SensorID's 39 and 49-53) and seven Raumedic sensors (SensorID's 46-48 and 54-57) were new while the other sensors had previously been used in patients. The proportion of ICP sensors with changes in baseline pressure $\geq 2 \mathrm{mmHg}$ is indicated in Table 1. 


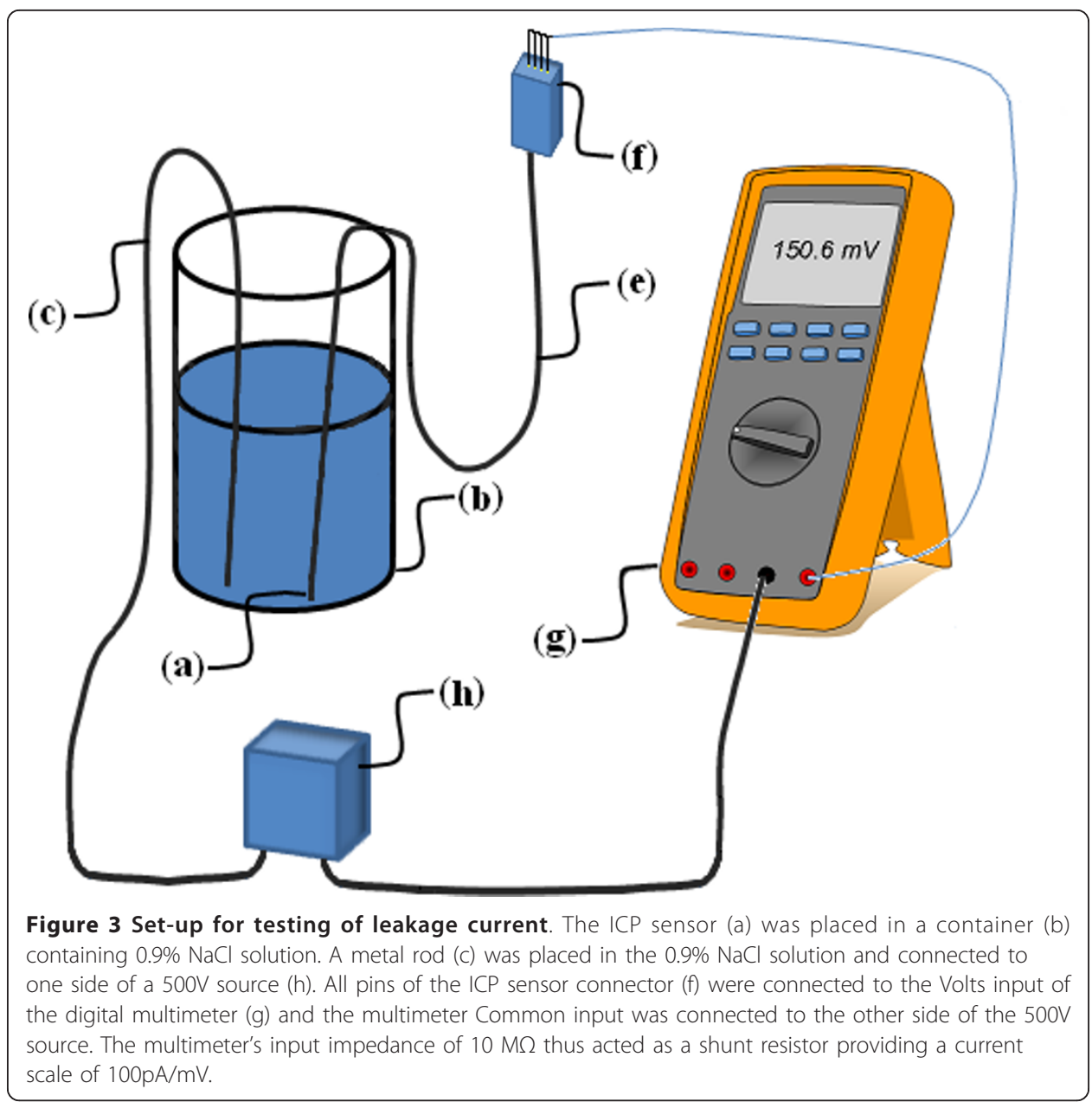

The Codman sensors typically responded with sudden shifts in baseline pressure, though gradual drifts in baseline pressure were seen in two sensors (SensorID's 7 and 37). The maximum lasting changes in baseline pressure of Codman sensors are shown in Table 2. Charging the test person to $5 \mathrm{kV}$ caused baseline shift in five sensors (SensorID's $37,38,40,41$ and 50 ), with a measured potential change of $2-5 \mathrm{kV}$ in the $0.9 \%$ $\mathrm{NaCl}$ solution with the sensor. In the other Codman sensors, changes in baseline pressure occurred when the test person was charged to a $10 \mathrm{kV}$ differential, in which potential changes to the sensor were comparable to that evoked when charging the person to $5 \mathrm{kV}$. For only one sensor (SensorID 38), we managed to deliver a $10 \mathrm{kV}$ potential change directly to the sensor (taking place after the sensor already had responded to $5 \mathrm{kV}$ ). Figure 4 illustrates shifts in baseline pressure in two sensors, and

Table 1 ICP sensors tested for altered baseline pressure following ESD

\begin{tabular}{ccc}
\hline Type of sensor & Number & Number (\%) with change baseline pressure $\mathbf{\geq} \mathbf{2} \mathbf{~ m m H g}$ \\
\hline Codman ICP Microsensor & 25 & $24(96)$ \\
Raumedic & & \\
NeuroVent P-C & 11 & $11(100)$ \\
NeuroVent P & 12 & $3(25)$ \\
NeuroDur & 9 & $3(33)$ \\
\hline
\end{tabular}


Table 2 Lasting alterations in baseline pressure of Codman sensors following ESD

\begin{tabular}{|c|c|c|c|c|}
\hline \multicolumn{2}{|c|}{ ICP Sensor } & \multicolumn{3}{|c|}{ Baseline pressure $(\mathrm{mmHg})$} \\
\hline Codman & SensorID & Before & After & Maximum change \\
\hline Microsensor & 1 & -0.7 & -13.3 & -12.6 \\
\hline$"$ & 2 & 0.2 & 13.4 & 13.2 \\
\hline$"$ & 3 & -1.5 & -9.5 & -8 \\
\hline$"$ & 4 & 1.7 & -24.4 & -26.1 \\
\hline$"$ & 5 & -0.02 & -1.2 & -1.18 \\
\hline$"$ & 6 & -0.2 & -5 & -4.8 \\
\hline$"$ & 7 & -0.2 & -17.7 & -17.5 \\
\hline$"$ & 8 & -0.6 & 8.7 & 9.3 \\
\hline$"$ & 9 & 11.5 & 3.8 & -7.7 \\
\hline$"$ & 20 & 0.5 & 9.5 & 9 \\
\hline$"$ & 36 & 0 & 4.8 & 4.8 \\
\hline$"$ & 37 & 8.8 & -5.3 & -14.1 \\
\hline$“$ & 38 & 0 & 23.2 & 23.2 \\
\hline$"$ & 39 & -0.7 & 2.8 & 3.5 \\
\hline$"$ & 40 & 0.4 & 40 & 39.6 \\
\hline$"$ & 41 & 0 & 16 & 16 \\
\hline$"$ & 42 & -6.3 & -26 & -19.7 \\
\hline$"$ & 43 & 1.8 & 13.1 & 11.3 \\
\hline$"$ & 44 & -9.3 & -0.1 & 9.2 \\
\hline$"$ & 45 & -4.3 & 0.02 & 4.32 \\
\hline$"$ & 49 & -12.2 & -23.1 & -10.9 \\
\hline$"$ & 50 & -0.1 & -15.9 & -15.8 \\
\hline$"$ & 51 & -15.3 & -0.8 & 14.5 \\
\hline$"$ & 52 & 4.9 & 12.5 & 7.6 \\
\hline$"$ & 53 & -0.4 & -8.6 & -8.2 \\
\hline
\end{tabular}

${ }^{1}$ Change in baseline pressure $\geq 2 \mathrm{mmHg}$ (absolute value) is highlighted.

gradual drift of baseline pressure in another. An animation of baseline shift subsequent to ESD's of SensorID 4 is shown in Additional File 3.

The Raumedic sensors responded differently to ESD's depending on the design of the sensors. While Raumedic NeuroVent P-C was highly unstable to ESD's, the Raumedic NeuroVent $\mathrm{P}$ and NeuroDur sensors differed. The maximum lasting changes in baseline pressure for the individual Raumedic sensors are presented in Table 3.

All the Raumedic NeuroVent P-C sensors responded with gradual drifts in their baseline pressure; drifts occurred when the test person was charged to $0.5 \mathrm{kV}$ in 3 sensors (SensorID's 16, 29 and 46), $2.5 \mathrm{kV}$ in 6 sensors (SensorID's 11-15, and 18), and 5 $\mathrm{kV}$ in two sensors (SensorID's 10 and 57). The gradual drift of NeuroVent P-C in SensorID 14 is shown in Figure 5a (see also Additional file 4).

The shifts in baseline pressure of NeuroVent $\mathrm{P}$ sensors were seen after charging the test person to $0.5 \mathrm{kV}$ in two (SensorID's 33 and 47), and to $2.5 \mathrm{kV}$ in another (SensorID 32). In Figure 5b is illustrated how SensorID 33 gradually changed its baseline pressure following repetitive ESD's of $0.5 \mathrm{kV}$ (an animation of the pressure signal is shown in Additional file 5).

All the three NeuroDur sensors responding to ESD (SensorID's 23, 25 and 28) responded when the test person was charged to $0.5 \mathrm{kV}$, with a corresponding $0.5 \mathrm{kV}$ potential change in the solution around the sensor. The responses of SensorID's 25 and 28 are shown in Figure 6 (see also Additional file 6). 
Eide and Bakken BioMedical Engineering OnLine 2011, 10:75

Page 7 of 16

http://www.biomedical-engineering-online.com/content/10/1/75
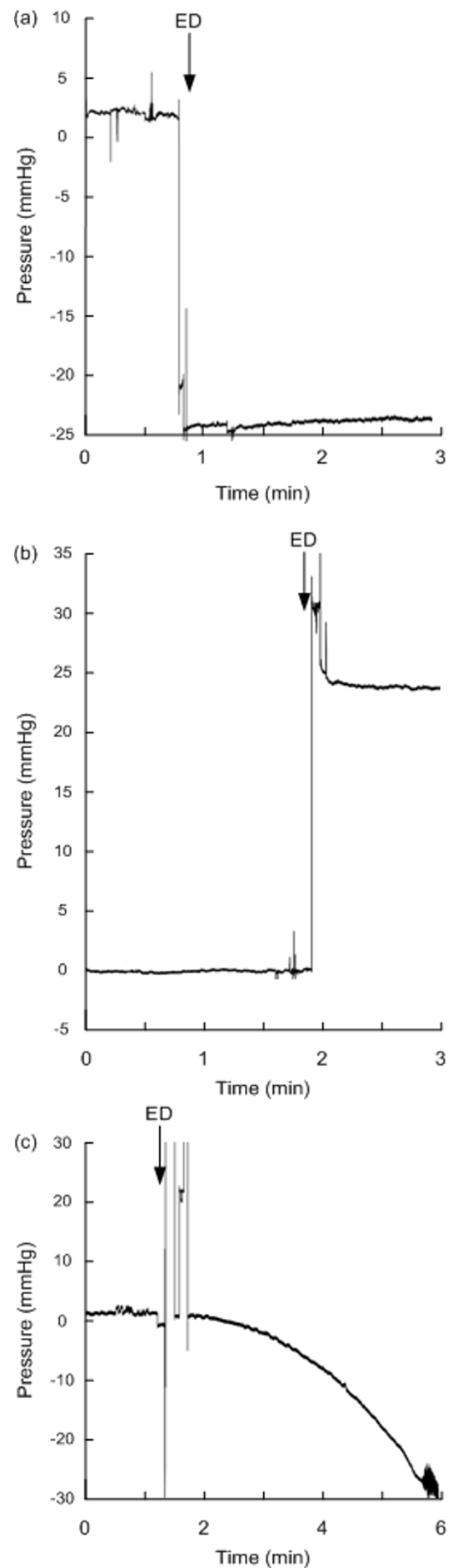

Figure 4 Continuous pressure signal from Codman sensors before/after electrostatic discharge. The continuous pressure signals retrieved from Codman Microsensor are shown before and after electrostatic 
discharges (ESD's) for SensorlD 4 (a), SensorlD 38 (b), and SensorlD 7 (c). Note that sudden changes in baseline pressure occurred for SensorlD's 4 and 38, while SensorlD 7 showed gradual drift of baseline pressure. The baseline pressure level $(\mathrm{mmHg})$ is indicated on the $y$ axis, and the time line on the $x$ axis levels; the ESD is indicated by an arrow.

\section{Experimental setup}

When the test-person was charged to $0.5 \mathrm{kV}$, the ESD delivered to the sensor was typically $0.5 \mathrm{kV}$ pulse peak. Charging to $5 \mathrm{kV}$ provided for a potential change of $2.5 \mathrm{kV}$ on average (range 2-5 kV). When charging to $10 \mathrm{kV}$, typically 3.5-4.0 kV change was seen; in a few occasions we observed even $7 \mathrm{kV}$ change (10 kV on one occasion). While ESD's $<3 \mathrm{kV}$ hardly provided any unpleasant sensations, ESD's of about $5 \mathrm{kV}$ gave weak unpleasant sensations, while ESD's $\geq 7 \mathrm{kV}$ provided a sensation which is evident, though not painful, to the test person.

Table 3 Lasting alterations in baseline pressure of Raumedic sensors following ESD

\begin{tabular}{|c|c|c|c|c|}
\hline \multicolumn{2}{|c|}{ ICP Sensor } & \multicolumn{3}{|c|}{ Baseline pressure $(\mathrm{mmHg})$} \\
\hline Raumedic & SensorID & Before & After & Maximum change $^{1}$ \\
\hline NeuroVent P-C & 10 & 18.4 & -9.3 & -27.7 \\
\hline$"$ & 11 & -0.3 & -11.5 & -11.2 \\
\hline$"$ & 12 & 9.5 & 3.6 & -5.9 \\
\hline$"$ & 13 & 0.6 & 6.9 & 6.3 \\
\hline$"$ & 14 & -5.4 & 11.4 & 16.8 \\
\hline$"$ & 15 & -0.7 & -14.1 & -13.4 \\
\hline$"$ & 16 & 0 & 18.3 & 18.3 \\
\hline$"$ & 18 & -1.4 & 1.1 & 2.5 \\
\hline$"$ & 29 & -5.7 & 17.3 & 23 \\
\hline “ & 46 & -12.1 & -0.1 & 12 \\
\hline$"$ & 57 & 1.3 & -8.3 & -9.6 \\
\hline NeuroVent $P$ & 17 & 1.6 & 0.1 & -1.5 \\
\hline$"$ & 30 & -0.2 & -0.5 & -0.3 \\
\hline$"$ & 31 & -0.05 & -0.9 & -0.85 \\
\hline$"$ & 32 & -0.3 & 4.7 & 5 \\
\hline$"$ & 33 & 0 & 9.6 & 9.6 \\
\hline$"$ & 34 & 0.2 & 0.7 & 0.5 \\
\hline$"$ & 35 & 0.1 & 0.5 & 0.4 \\
\hline$"$ & 47 & 2.5 & -0.1 & -2.6 \\
\hline$"$ & 48 & 0.4 & -0.1 & -0.5 \\
\hline$"$ & 54 & 0.6 & -0.9 & -1.5 \\
\hline$"$ & 55 & 0.3 & 0.5 & 0.2 \\
\hline$"$ & 56 & 0 & -0.1 & -0.1 \\
\hline NeuroDur & 19 & 0.1 & -0.6 & -0.7 \\
\hline$"$ & 21 & -0.05 & -0.1 & -0.05 \\
\hline$"$ & 22 & 0 & 0.1 & 0.1 \\
\hline$"$ & 23 & 52.4 & 0.8 & -51.6 \\
\hline$"$ & 24 & 0.8 & 0.9 & 0.1 \\
\hline$"$ & 25 & 0.4 & 10.7 & 10.3 \\
\hline$"$ & 26 & 0.8 & 0.3 & -0.5 \\
\hline$"$ & 27 & 0.1 & 0.2 & 0.1 \\
\hline$"$ & 28 & 0 & 39 & 39 \\
\hline
\end{tabular}

${ }^{1}$ Change in baseline pressure $\geq 2 \mathrm{mmHg}$ (absolute value) is highlighted. 


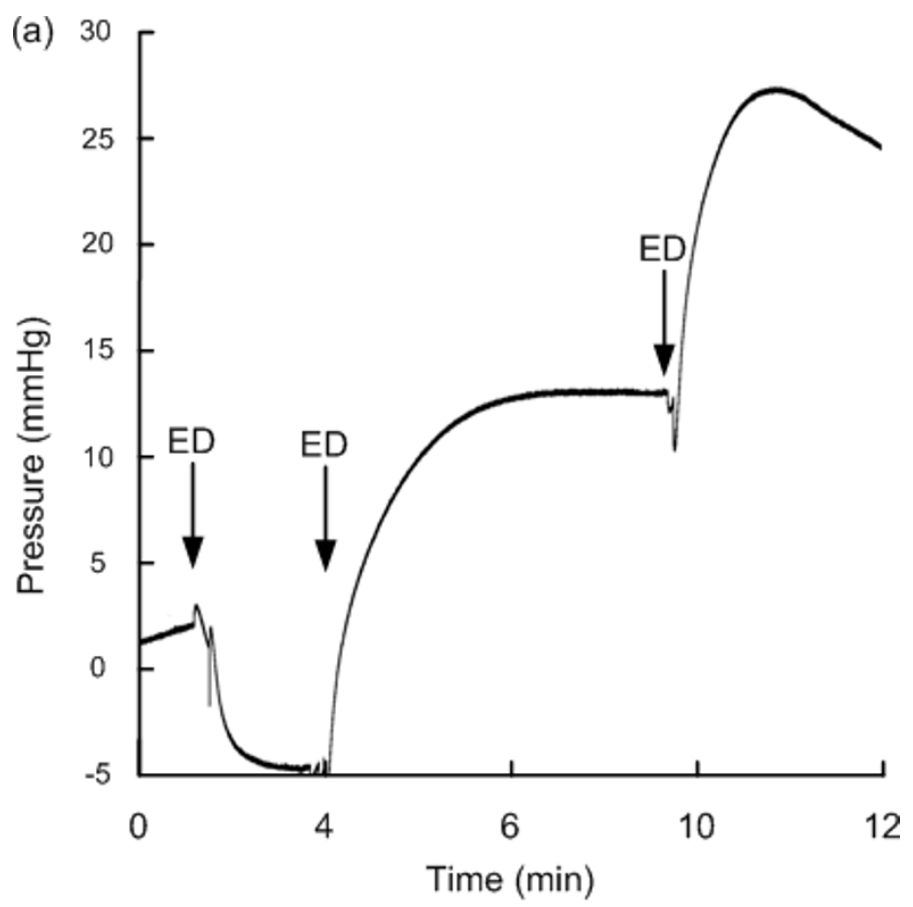

(b)

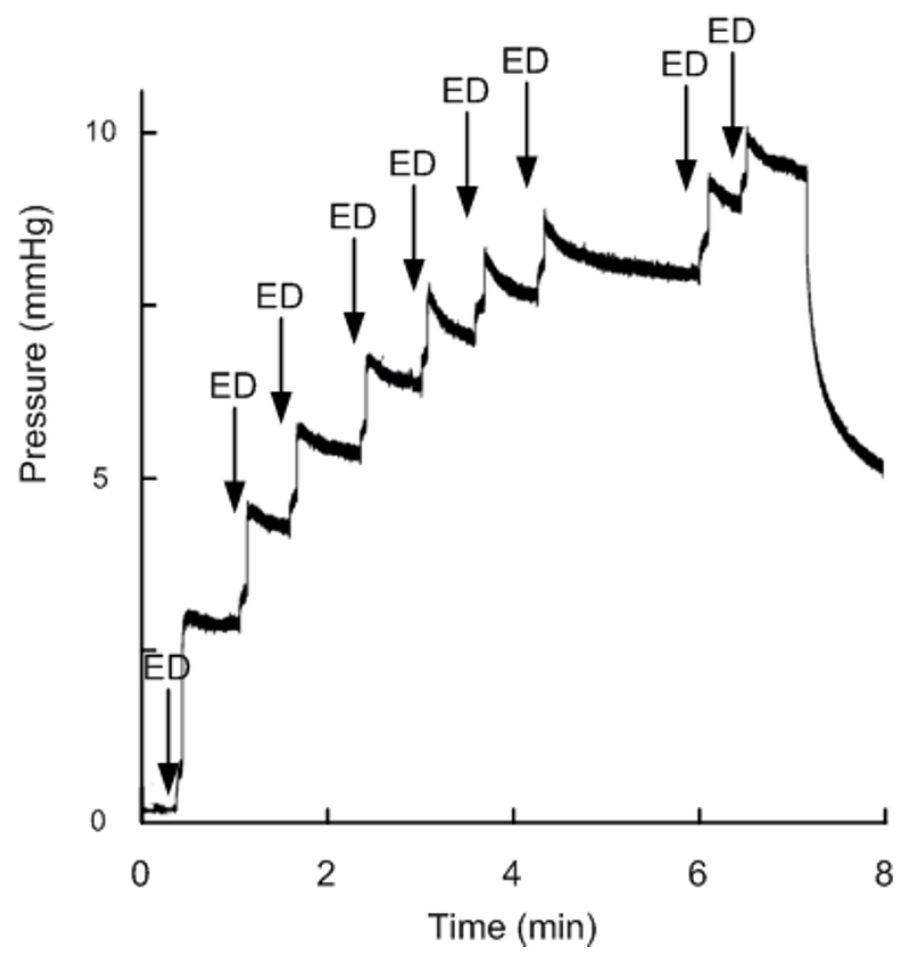

Figure 5 Continuous pressure signal from Raumedic sensors before/after electrostatic discharge. The continuous pressure signals retrieved from Raumedic NeuroVent sensors are shown before and after electrostatic discharges (ESD's) for SensorlD 14 (NeuroVent P-C; a), and for SensorlD 33 (NeuroVent P; b). The baseline pressure level $(\mathrm{mmHg})$ is indicated on the $y$ axis, and the time line on the $\times$ axis levels; the ESD is indicated by an arrow. Note that gradual drifts in baseline pressure occurred. For SensorlD 33, repeated low ESD's of $0.5 \mathrm{kV}$ (not being sensed by the test person) causes gradual build-up of baseline pressures. 

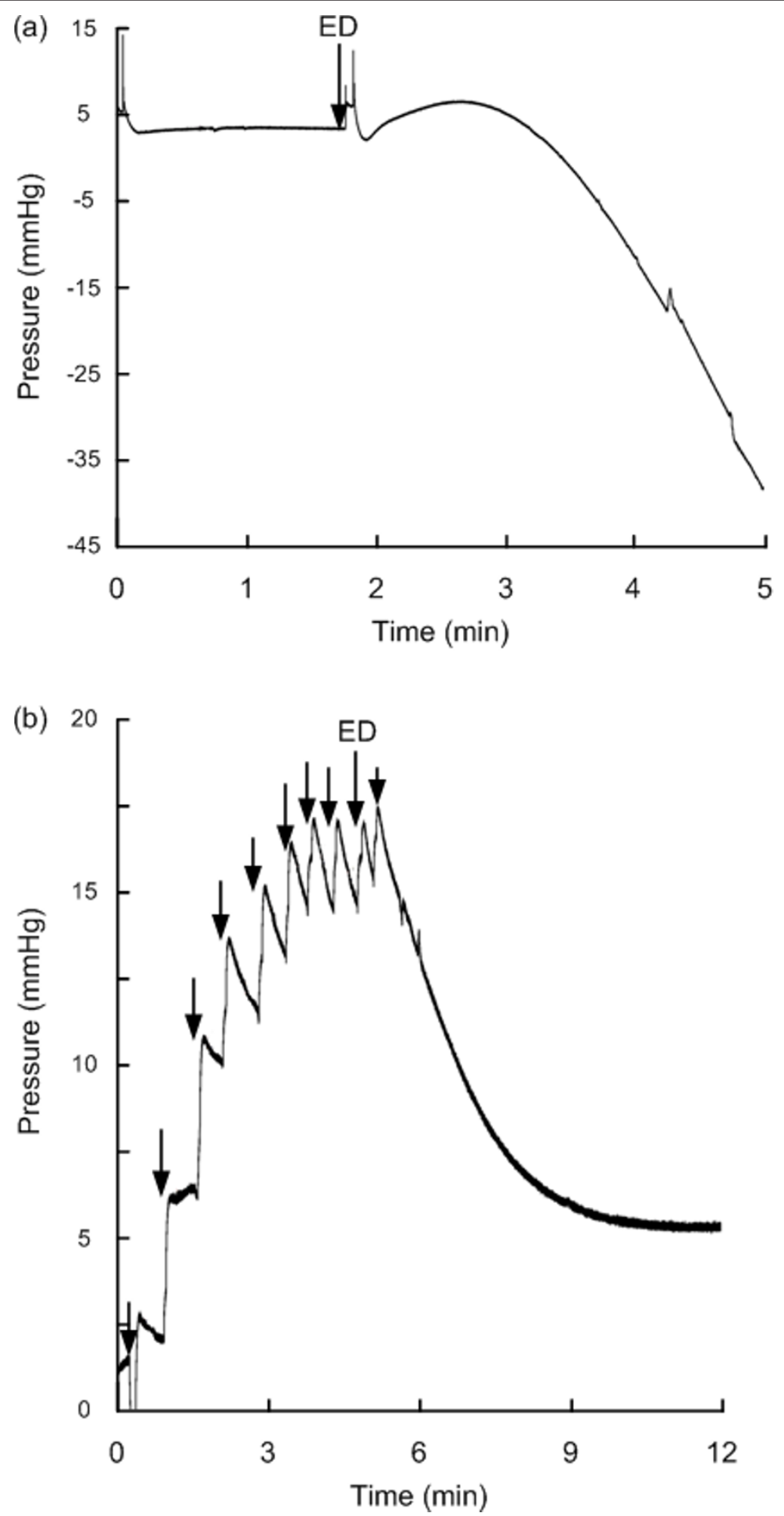

Figure 6 Continuous pressure signal from Raumedic sensors before/after electrostatic discharge The continuous pressure signals retrieved from Raumedic NeuroDur sensors are shown before and after electrostatic discharges (ESD's) of SensorlD 28 (a), and for SensorlD 25 (b). The baseline pressure level $(\mathrm{mmHg})$ is indicated on the $y$ axis, and the time line on the $\times$ axis levels; the ESD is indicated by an arrow. Note that gradual drifts in baseline pressure occurred. 


\section{Leakage current}

The results of testing leakage current are presented in Table 4. Leakage current was seen in 1 of the 25 Codman sensors (SensorID 45); this sensor revealed leakage current of up to100 nA with continuous breakthrough.

The Raumedic sensors differed depending on design. The NeuroVent P-C sensors showed a marginally higher leakage current. Two of the NeuroVent P sensors responding to ESD's (SensorID's 32 and 33) showed leakage current. The three NeuroDur sensors (SensorID's 23, 25 and 28) being affected by ESD's of $0.5 \mathrm{kV}$, all showed abnormal current leakage.

\section{Discussion}

This study shows that the baseline pressures (zero point) of Codman and Raumedic ICP sensors can be altered by ESD's at magnitudes that are clinically relevant. The observations indicate severe limitations with currently used ICP sensors.

\section{ICP sensors used for clinical monitoring of ICP}

The ICP sensors tested in this study are widely used ICP sensors. Both the Codman $[10,14,16,21,22,25,27]$ and Raumedic $[12,19,29,30]$ sensors have undergone extensive bench and clinical testing. In general, the assessment of ICP sensors has previously focused on long-term-drift of the sensors, sensitivity to temperature changes, and inter-sensor accuracy comparisons [10,11,13-19,22,25].

All the ICP sensors measure pressure relative to atmospheric pressure, which means that they have to be zeroed before measuring ICP. Hence, their zero point equates the atmospheric pressure, and the ICP level displayed on the monitor represents the difference between pressure level within the intracranial compartment and the sensor zero point. It should be noted that in daily clinical practice, various notations are used to refer to the zero point, such as set point, reference pressure, or baseline pressure. In this paper and previous publications [30-33], we have preferred the term baseline pressure, when referring to the zero point of the ICP sensor.

Depending on clinical state, the upper normal threshold of ICP varies between 15 and $25 \mathrm{mmHg}$ [1-4]. Obviously, if the baseline pressure (zero point) spontaneously shifts $>10-20 \mathrm{mmHg}$, the ICP presented to the physician becomes wrong. Since the continuous monitoring of ICP is done for surveillance of patients with brain injury, e. g. due to traumatic brain injury, stroke complications to brain surgery [1-3], false ICP values represent a likely hazard to the patient. For example, when ICP increases, efforts may be done to reduce the ICP; such efforts include medication, artificial ventilation and surgical procedures.

When the impact of ESD's on ICP sensors previously has not been considered, the reason may be that the issues of non-physiological changes in baseline pressure have not been regarded as a problem in ICP monitoring.

\section{Electrostatic discharges in the hospital environment}

There are different ways to test ESD's; it has also been addressed that there is a need for more standardized methods [40]. The rational for our experimental setup was to best possible test ESD's of clinically relevant magnitudes. Therefore, the ESD's were delivered from a test person, and caused pulse peak discharges to the sensor typically 
Table 4 Results of testing of current leakage

\begin{tabular}{|c|c|c|c|c|}
\hline \multicolumn{2}{|c|}{ ICP sensor } & \multicolumn{3}{|c|}{ Test parameters } \\
\hline Type & ${ }^{\mathrm{a}}$ SensorlD & Test Voltage & Leakage Current & Resistance \\
\hline Codman Microsensor & 1 & $500 \mathrm{~V}$ & $70 \mathrm{pA}$ & $7 \mathrm{~T} \Omega$ \\
\hline Codman Microsensor & 2 & $500 \mathrm{~V}$ & $80 \mathrm{pA}$ & $6 \mathrm{~T} \Omega$ \\
\hline Codman Microsensor & 3 & $500 \mathrm{~V}$ & $130 \mathrm{pA}$ & $4 \mathrm{~T} \Omega$ \\
\hline Codman Microsensor & 4 & $500 \mathrm{~V}$ & $60 \mathrm{pA}$ & $8 \mathrm{~T} \Omega$ \\
\hline Codman Microsensor & 5 & $500 \mathrm{~V}$ & $60 \mathrm{pA}$ & $8 \mathrm{~T} \Omega$ \\
\hline Codman Microsensor & 6 & $500 \mathrm{~V}$ & $100 \mathrm{pA}$ & $5 \mathrm{~T} \Omega$ \\
\hline Codman Microsensor & 7 & $500 \mathrm{~V}$ & $60 \mathrm{pA}$ & $8 \mathrm{~T} \Omega$ \\
\hline Codman Microsensor & 8 & $500 \mathrm{~V}$ & $90 \mathrm{pA}$ & $6 \mathrm{~T} \Omega$ \\
\hline Codman Microsensor & 9 & $500 \mathrm{~V}$ & $50 \mathrm{pA}$ & $10 \mathrm{~T} \Omega$ \\
\hline Raumedic NeuroVent P-C & 10 & $500 \mathrm{~V}$ & $150 \mathrm{pA}$ & $3 T \Omega$ \\
\hline Raumedic NeuroVent P-C & 11 & $500 \mathrm{~V}$ & $140 \mathrm{pA}$ & $4 \mathrm{~T} \Omega$ \\
\hline Raumedic NeuroVent P-C & 12 & $500 \mathrm{~V}$ & $110 \mathrm{pA}$ & $5 \mathrm{~T} \Omega$ \\
\hline Raumedic NeuroVent P-C & 13 & $500 \mathrm{~V}$ & $270 \mathrm{pA}$ & $1.9 \mathrm{~T} \Omega$ \\
\hline Raumedic NeuroVent P-C & 14 & $500 \mathrm{~V}$ & $100 \mathrm{pA}$ & $5 \mathrm{~T} \Omega$ \\
\hline Raumedic NeuroVent P-C & 15 & $500 \mathrm{~V}$ & $150 \mathrm{pA}$ & $3 T \Omega$ \\
\hline Raumedic NeuroVent P-C & 16 & $500 \mathrm{~V}$ & 290 pA & $1.7 \mathrm{~T} \Omega$ \\
\hline Raumedic NeuroVent P & 17 & $143 \mathrm{~V}$ & $27.6 \mu \mathrm{A}$ & $5.2 \mathrm{M} \Omega$ \\
\hline Raumedic NeuroVent P-C & 18 & $500 \mathrm{~V}$ & $300 \mathrm{pA}$ & $1.5 \mathrm{~T} \Omega$ \\
\hline Raumedic NeuroDur & 19 & $142 \mathrm{~V}$ & $27.6 \mu \mathrm{A}$ & $5.1 \mathrm{M} \Omega$ \\
\hline Codman Microsensor & 20 & $500 \mathrm{~V}$ & $50 \mathrm{pA}$ & $10 \mathrm{~T} \Omega$ \\
\hline Raumedic NeuroDur & 21 & $500 \mathrm{~V}$ & - & $22 \Omega$ \\
\hline Raumedic NeuroDur & 22 & $142 \mathrm{~V}$ & $27.6 \mu \mathrm{A}$ & $5.1 \mathrm{M} \Omega$ \\
\hline Raumedic NeuroDur & 23 & $273 \mathrm{~V}$ & $\sim 16 \mu \mathrm{A}$ & $\sim 17 \mathrm{M} \Omega$ \\
\hline Raumedic NeuroDur & 24 & $142 \mathrm{~V}$ & $27.6 \mu \mathrm{A}$ & $5.1 \mathrm{M} \Omega$ \\
\hline Raumedic NeuroDur & 25 & $351 \mathrm{~V}$ & $9.07 \mu \mathrm{A}$ & $38.7 \mathrm{M} \Omega$ \\
\hline Raumedic NeuroDur & 26 & $142 \mathrm{~V}$ & $27.6 \mu \mathrm{A}$ & $5.1 \mathrm{M} \Omega$ \\
\hline Raumedic NeuroDur & 27 & $142 \mathrm{~V}$ & $27.6 \mu \mathrm{A}$ & $5.1 \mathrm{M} \Omega$ \\
\hline Raumedic NeuroDur & 28 & $360 \mathrm{~V}$ & $8 \mu \mathrm{A}$ & $45 \mathrm{M} \Omega$ \\
\hline Raumedic NeuroVent P-C & 29 & $500 \mathrm{~V}$ & $130 \mathrm{pA}$ & $4 \mathrm{~T} \Omega$ \\
\hline Raumedic NeuroVent P & 30 & $142 \mathrm{~V}$ & $27.6 \mu \mathrm{A}$ & $5.1 \mathrm{M} \Omega$ \\
\hline Raumedic NeuroVent $P$ & 31 & $143 \mathrm{~V}$ & $27.6 \mu \mathrm{A}$ & $5.2 \mathrm{M} \Omega$ \\
\hline Raumedic NeuroVent P & 32 & $335 \mathrm{~V}$ & $10.5 \mu \mathrm{A}$ & $32 \mathrm{M} \Omega$ \\
\hline Raumedic NeuroVent P & 33 & $500 \mathrm{~V}$ & $200 \mathrm{pA}$ & $2.5 \mathrm{~T} \Omega$ \\
\hline Raumedic NeuroVent P & 34 & $273 \mathrm{~V}$ & $\sim 16 \mu \mathrm{A}$ & $\sim 17 \mathrm{M} \Omega$ \\
\hline Raumedic NeuroVent P & 35 & $500 \mathrm{~V}$ & - & $100 \Omega$ \\
\hline Codman Microsensor & 36 & $500 \mathrm{~V}$ & $80 \mathrm{pA}$ & $6 \mathrm{~T} \Omega$ \\
\hline Codman Microsensor & 37 & $500 \mathrm{~V}$ & $70 \mathrm{pA}$ & $7 \mathrm{~T} \Omega$ \\
\hline Codman Microsensor & 38 & $500 \mathrm{~V}$ & $70 \mathrm{pA}$ & $7 \mathrm{~T} \Omega$ \\
\hline Codman Microsensor & 39 & $500 \mathrm{~V}$ & $70 \mathrm{pA}$ & $7 \mathrm{~T} \Omega$ \\
\hline Codman Microsensor & 40 & $500 \mathrm{~V}$ & $50 \mathrm{pA}$ & $10 \mathrm{~T} \Omega$ \\
\hline Codman Microsensor & 41 & $500 \mathrm{~V}$ & $90 \mathrm{pA}$ & $6 \mathrm{~T} \Omega$ \\
\hline Codman Microsensor & 42 & $500 \mathrm{~V}$ & $90 \mathrm{pA}$ & $6 \mathrm{~T} \Omega$ \\
\hline Codman Microsensor & 43 & $500 \mathrm{~V}$ & $80 \mathrm{pA}$ & $6 \mathrm{~T} \Omega$ \\
\hline Codman Microsensor & 44 & $500 \mathrm{~V}$ & $60 \mathrm{pA}$ & $8 \mathrm{~T} \Omega$ \\
\hline Codman Microsensor & 45 & $500 \mathrm{~V}$ & $0.5 \sim 100 \mathrm{nA}$ & $\sim$ \\
\hline Raumedic NeuroVent P-C & 46 & $500 \mathrm{~V}$ & $210 \mathrm{pA}$ & $2.4 \mathrm{~T} \Omega$ \\
\hline Raumedic NeuroVent P & 47 & $142 \mathrm{~V}$ & $27.5 \mu \mathrm{A}$ & $5.2 \mathrm{M} \Omega$ \\
\hline Raumedic NeuroVent P & 48 & $142 \mathrm{~V}$ & $27.5 \mu \mathrm{A}$ & $5.2 \mathrm{M} \Omega$ \\
\hline Codman Microsensor & 49 & $500 \mathrm{~V}$ & $100 \mathrm{pA}$ & $5 \mathrm{~T} \Omega$ \\
\hline Codman Microsensor & 50 & $500 \mathrm{~V}$ & $210 \mathrm{pA}$ & $2.4 \mathrm{~T} \Omega$ \\
\hline
\end{tabular}


Table 4 Results of testing of current leakage (Continued)

\begin{tabular}{llllc}
\hline Codman Microsensor & $\mathbf{5 1}$ & $500 \mathrm{~V}$ & $130 \mathrm{pA}$ & $4 \mathrm{~T} \Omega$ \\
Codman Microsensor & $\mathbf{5 2}$ & $500 \mathrm{~V}$ & $100 \mathrm{pA}$ & $5 \mathrm{~T} \Omega$ \\
Codman Microsensor & $\mathbf{5 3}$ & $500 \mathrm{~V}$ & $120 \mathrm{pA}$ & $4 \mathrm{~T} \Omega$ \\
Raumedic NeuroVent P & $\mathbf{5 4}$ & $143 \mathrm{~V}$ & $27.6 \mu \mathrm{A}$ & $5.2 \mathrm{M} \Omega$ \\
Raumedic NeuroVent P & $\mathbf{5 5}$ & $143 \mathrm{~V}$ & $27.6 \mu \mathrm{A}$ & $5.2 \mathrm{M} \Omega$ \\
Raumedic NeuroVent P & $\mathbf{5 6}$ & $143 \mathrm{~V}$ & $27.6 \mu \mathrm{A}$ & $5.2 \mathrm{M} \Omega$ \\
Raumedic NeuroVent P-C & $\mathbf{5 7}$ & $500 \mathrm{~V}$ & $320 \mathrm{pA}$ & $1.6 \mathrm{~T} \Omega$ \\
\hline
\end{tabular}

SensorlDa: The SensorlD's of new and previously non-used sensors are presented with numbers in bold.

in the range $0.5-5 \mathrm{kV}$. Such ESD's may not be unpleasant to the test person, and are below the levels that can be seen clinically [39,41]. ESD's of magnitudes $<2-3 \mathrm{kV}$ may not even be appreciated by the personnel taking care of the patients. It was previously demonstrated that potentials $>30 \mathrm{kV}$ could be induced on the bed framework when the bedding is pulled from the bed; the degree of charging being dependent on the material of hospital bedding [39]. In comparison, previous tests in our hospital showed that ESD's of 20-40 kV could be seen, depending on the textiles used in clothing (Jensen, Grimnes, unpublished data). Using the test approach described here, we avoided ESD's of magnitudes that are not clinically relevant. Only in a few instances, we managed to deliver $7 \mathrm{kV}$ potential changes to the sensor $(10 \mathrm{kV}$ in one sensor that first responded markedly to $5 \mathrm{kV}$ ). Accordingly, the voltages referred to here are quite low.

\section{Different characteristics of Codman and Raumedic ICP sensors}

There were some differences between the Codman and Raumedic sensors in their responses to ESD's. The Codman sensors consistently responded to electrostatic changes of 2-3 kV, with sudden shifts in baseline pressure. Gradual drift was only seen in 2 of 25 Codman sensors (8\%). These findings compare with our clinical observations of spontaneous alterations in baseline ICP despite unchanged ICP waveform. The observation that baseline pressure was changed maximally $>10 \mathrm{mmHg}$ in 13 of 25 (52\%) sensors (and > $20 \mathrm{mmHg}$ in 3 (12\%) sensors), indicate that effects of ESD's are of a magnitude that likely would affect patient management.

The Raumedic sensors responded differently depending on their design. Two types of responses were seen, namely gradual drifts and sudden shifts in baseline pressure. While the NeuroVent P-C was completely unstable to ESD's, even at levels of $0.5 \mathrm{kV}$, the NeuroVent $\mathrm{P}$ was less affected. The P-C type incorporates a ceramic coating on the sensor tip while the P type uses titanium. Also the NeuroDur sensor using titanium was more stable, where the tip seemed connected to the sensor with a $5 \mathrm{M} \Omega$ resistance. Nevertheless, the observation of alterations in baseline pressure $>10 \mathrm{mmHg}$ in 10 of 32 (31\%) Raumedic sensors (> $20 \mathrm{mmHg}$ in 4 of $32(12.5 \%)$ indicate that the effects of ESD's would affect patient management also when using these sensors. In a recent study comparing simultaneous ICP signals from Raumedic NeuroVent and NeuroDur sensors, we encountered average differences between sensors during over-night monitoring > $10 \mathrm{mmHg}$ in 4 of 12 (33\%) patients [30].

While leakage current was seen in only one Codman sensor, and no Raumedic NeuroVent P-C sensors, current leakage was seen in 2 of 3 Raumedic NeuroVent P sensors that responded to ESD's, and in all three Raumedic NeuroDur sensors responding to ESD's. The testing of leakage current indicated that in Raumedic titanium sensors 
(NeuroVent $\mathrm{P}$ and NeuroDur) there is an internal $5 \mathrm{M} \Omega$ resistance between the metal shell and the connector. Hence, the sensors with resistance different from $5 \mathrm{M} \Omega$ (Table 4) might have a broken protection resistor. We found, however, no evidence of sensor damage using microscopy, though damage to ICP sensors may happen both during the implantation and explanation.

\section{Control of risk associated with ESD's}

A major issue with both the Codman and Raumedic ICP sensors is that the health care personnel get no warning about sudden shifts in baseline pressure (zero point) of ICP sensors, or even damage to the ICP sensor during/after implantation. Thereby it is impossible for the physician or nurse to know whether changes in ICP are related to ESD's or not. The Codman sensor cannot be re-zeroed because this is done within the operating room before sensor implantation. The Raumedic sensors, on the other hand, can be re-zeroed after implantation; however, this procedure is not necessarily done by the nurse/physician when ICP is changing.

While the present study focused on effects of ESD's on ICP sensors, the baseline pressure can also be affected by user-related wrong zeroing or even damage to the sensor during implantation, which may not be recognized. Therefore, it can be questioned why modern monitoring systems include no warning. Such warning should be incorporated as part of risk control.

We suggest that a robust way of incorporating risk control is by determining the ICP from the ICP waveform itself. Thereby quality control is accomplished and the issue of baseline pressure alterations is eliminated. The first author previously described a procedure for automatic identification of the cardiac-induced waves in the ICP waveform [42]. Using this approach, the ICP parameters such as the mean ICP wave amplitude (MWA), can be determined from the cardiac induced ICP waves [42]. Since such determination of single wave pressure parameters is done within the ICP signal itself, the analysis results is not affected by changes in baseline pressure. The automatic identification of verified cardiac induced ICP waves also recognizes other ICP sensor-related issues. For example, if an ICP sensor is placed wrong by mistake, artificial waves and no cardiac induced ICP waves will be identified, providing feedback to the user that the ICP signal is erroneous.

\section{Conclusions}

The baseline pressure (zero point) of the Codman and Raumedic ICP sensors can be altered by ESD's at discharge magnitudes that are clinically relevant levels. The shifts in baseline pressure will directly affect the ICP levels visualised to the health care personnel. The alterations in baseline pressure can be extensive (>10-20 $\mathrm{mmHg}$ ), thereby revealing wrong ICP values, which subsequently poses a high risk for erroneous treatment.

\section{Additional material}

Additional file 1: The testing of a Codman sensor. The video shows the testing of a Codman sensor. A test person is being charged, and then touches the metal rod, leading the electrostatic discharge to the container filled with $0.9 \% \mathrm{NaCl}$ Ringer solution, wherein the sensor is placed. A schematic illustration is shown in Figure 2.

Additional file 2: The testing of a Raumedic sensor. The video shows the testing of a Raumedic sensor. A test person is being charged, and then touches the metal rod, leading the electrostatic discharge to the container filled with $0.9 \% \mathrm{NaCl}$ Ringer solution, wherein the sensor is placed. A schematic illustration is shown in Figure 2. 
Additional file 3: A continuous pressure signal before and after ESD - Codman sensor. The continuous pressure signal of a Codman sensor (SensorlD 4) is shown at a higher velocity (about $\times 30$ ) than normal. The test person was charged to $10 \mathrm{kV}$, which delivered an ESD of $5 \mathrm{kV}$ to the sensor. At the time of the ESD, a sudden change in baseline pressure occurs. See also Figure 4a.

Additional file 4: A continuous pressure signal before and after ESD - Raumedic NeuroVent P-C sensor The continuous pressure signal of a Raumedic P-C sensor (SensorlD 14) is shown at a higher velocity (about $\times 30$ ) than normal. The test person was charged to $2.5 \mathrm{kV}$ three times, which delivered ESD's of 1-2.5 kV to the sensor. At the time of the first ESD, a slight reduction of baseline pressure occurred, while at the second and third ESD's, the baseline pressures drifted to higher levels. See also Figure $5 \mathrm{a}$.

Additional file 5: A continuous pressure signal before and after ESD - Raumedic NeuroVent P sensor. The continuous pressure signal of a Raumedic $P$ sensor (SensorlD 33) is shown at a higher velocity (about $\times 30$ ) than normal. The test person was repeatedly charged to $0.5 \mathrm{kV}$, which delivered ESD's of $0.5 \mathrm{kV}$ to the sensor. At the time of each ESD, a slight increase of baseline pressure occurred. See also Figure $5 \mathrm{~b}$.

Additional file 6: A continuous pressure signal before and after ESD - Raumedic NeuroDur sensor. The continuous pressure signal of a Raumedic NeuroDur sensor (SensorlD 28) is shown at a higher velocity (about $X$ 30) than normal. The test person was charged to $0.5 \mathrm{kV}$, which delivered an ESD of $0.5 \mathrm{kV}$ to the sensor. At the time of ESD, a marked downward drift of baseline pressure occurred. See also Figure $6 a$.

\section{Abbreviations}

ICP: Intracranial pressure; ESD: Electrical discharge; kV: kilo Volt; CSF: cerebrospinal fluid; MWA: mean ICP wave amplitude; SW: single wave.

\section{Author details}

${ }^{1}$ Department of Neurosurgery, Oslo University Hospital, Rikshospitalet, Oslo, Norway. ${ }^{2}$ Faculty of Medicine, University of Oslo, Oslo, Norway. ${ }^{3}$ Department of Clinical and Biomedical Engineering, Oslo University Hospital, Rikshospitalet, Oslo, Norway.

\section{Authors' contributions}

Both authors have made substantial contributions to conception and design, acquisition of data, analysis and interpretation of data; and have been involved in drafting the manuscript or revising it critically for important intellectual content. Both authors have read and approved the final manuscript.

\section{Competing interests}

$A B$ reports no conflicts of interest. PKE has financial interest in the software company (dPCom A/S) that manufactures the software (Sensometrics ${ }^{\oplus}$ Research software and Sensometrics ${ }^{\oplus}$ Software), which was used for digital recording of the continuous pressure signals in this study.

Received: 29 March 2011 Accepted: 22 August 2011 Published: 22 August 2011

\section{References}

1. Marmarou A, Anderson RL, Ward JD, et al: Impact of ICP instability and hypotension on outcome in patients with severe head trauma. J Neurosurg 1991, 75:S59-S66.

2. Ghajar J: Traumatic brain injury. The Lancet 2000, 356:923-929.

3. Czosnyka M, Pickard JD: Monitoring and interpretation of intracranial pressure. J Neurol Neurosurg Psychiatry 2004, 75:813-821.

4. Padayachy $L C$, Figaji $A A$, Bullock MR: Intracranial pressure monitoring for traumatic brain injury in the modern era. Child's Nerv Syst 2010, 26:441-452.

5. Janny P: La pression intracrannienne Chez l'Homme Paris, Thesis; 1950.

6. Lundberg N: Continuous recording and control of ventricular fluid pressure in neurosurgical practice. Acta Psychiatr Neurol Scand 1960, 36:1-193.

7. Allen R: Intracranial pressure: A review of clinical problems, measurement techniques and monitoring methods. Med Engin Tech 1986, 10:299-320.

8. Zhong J, Dujovny M, Park HK, et al: Advances in ICP monitoring techniques. Neurol Res 2003, 25:339-350.

9. Gambardella G, d'Avella D, Tomasello F: Monitoring of brain tissue pressure with a fiberoptic device. Neurosurgery 1992, 31:918-922.

10. Piper IR, Miller JD: The evaluation of the wave-form analysis capability of a new strain-gauge intracranial pressure microsensor. Neurosurgery 1995, 36:1142-1145.

11. Yau YH, Piper I, Clutton RE Whittle IR: Experimental evaluation of the Spiegelberg intracranial pressure and intracranial compliance monitor. J Neurosurg 2000, 93:1072-1077.

12. Stendel R, Heidenreich J, Schilling A, et al: Clinical evaluation of a new intracranial pressure monitoring device. Acta Neurochir 2003, 145:185-193.

13. Allin D, Czosnyka M, Czosnyka Z: Laboratory testing of the Pressio intracranial pressure monitor. Neurosurgery 2008, 62:1158-1161.

14. Czosnyka M, Czosnyka Z, Pickard JD: Laboratory testing of three intracranial pressure microtransducers: Technical report. Neurosurgery 1996, 38:219-224.

15. Morgalla MH, Mettenleiter $\mathrm{H}$, Bitzer M, et al: ICP measurement control: Laboratory test of 7 types of intracranial pressure transducers. J Medical Engineer Techn 1999, 23:144-151. 
16. Morgalla MH, Krasznai L, Dietz K, et al: Methods of experimental and clinical assessment of the relative measurement accuracy of an intracranial pressure transducer. Technical note. J Neurosurg 2001, 95:529-532.

17. Morgalla MH, Dietz K, Deininger M, et al: The problem of long-term ICP drift assessment: Improvement by use of the ICP drift index. Acta Neurochir 2002, 144:57-61.

18. Piper I, Barnes A, Smith D, et al: The Camino intracranial pressure sensor: Is it optimal technology? An internal audit with a review of current intracranial pressure monitoring technologies. Neurosurgery 2001, 49:1158-1165.

19. Citerio G, Piper I, Cormio M, et al: Bench test assessment of the new Raumedic Neurovent-P ICP sensor: a technical report by the Brain-IT group. Acta Neurochir (Wien) 2004, 146:1221-1226.

20. Pople IK, Muhlbauer MS, Sanford RA, et al: Results and complications of intracranial pressure monitroing in 303 children. Pediatr Neurosurg 1995, 23:64-67.

21. Gray WP, Palmer JD, Gill J, et al: A clinical study of parenchymal and subdural miniature strain-gauge transducers for monitoring intracranial pressure. Neurosurgery 1996, 39:927-932.

22. Fernandes HM, Bingham K, Chambers IR, Mendelow AD: Clinical evaluation of the Codman microsensor inracranial pressure monitoring system. Acta Neurochir 1998, 71(Suppl):44-46.

23. Guyot LL, Dowling C, Diaz FG, et al: Cerebral monitoring devices: Analysis of complications. Acta Neurochir 1998, 71(Suppl):47-49

24. Rossi S, Buzzi F, Paparella A, et al: Complications and safety associated with ICP monitoring: A study of 542 patients. Acta Neurochir 1998, 71(Suppl):91-93.

25. Signorini DF, Shad A, Piper IR, et al: A clinical evaluation of the Codman Microsensor for intracranial pressure monitoring. Br J Neurosurg 1998, 1:223-227.

26. Lang J-M, Beck J, Zimmermann M: Clinical evaluation of intraparenchymal Spiegelberg pressure sensor. Neurosurgery 2003, 52:1455-1459.

27. Koskinen L-O, Olivecrona M: Clinical experience with the intraparenchymal intracranial pressure monitoring Codman Microsensor system. Neurosurgery 2005, 56:693-698.

28. Gelabert-Gonzàlez M, Ginesta-Galan V, Sernamito-Garcia R, et al: The Camino intracranial pressure device in clinical practice. Assessment in a 1000 cases. Acta Neurochir 2006, 148:435-441.

29. Poca M, Martinez-Ricarte F, Sahuquillo J, et al: Intracranial pressure monitoring with the Neurodur-P epidural sensor: A prospective study in patients with adult hydrocephalus or idiopathic intracranial hypertension. J Neurosurg 2008, 108:934-942.

30. Eide PK, Sorteberg W: Simultaneous measurements of intracranial pressure parameters in the epidural space and in brain parenchyma in patients with hydrocephalus. J Neurosurg 2010, 113:1317-1325.

31. Eide PK: Comparison of simultaneous continuous intracranial pressure (ICP) signals from a Codman and a Camino ICP sensor. Med Eng Physics 2006, 28:542-549.

32. Eide PK: Comparison of simultaneous continuous intracranial pressure (ICP) signals from ICP sensors placed within the brain parenchyma and the epidural space. Med Eng Physics 2008, 30:34-40.

33. Eide PK, Rapoport Bl, Gormley WB, et al: A dynamic nonlinear relationship between the static and pulsatile components of intracranial pressure in patients with subarachnoid hemorrhage. J Neurosurg 2010, 112:616-625.

34. Bruner JM: Hazards of electrical apparatus. Anesthesiology 1967, 28:396-425

35. Bruner JM, Aronow S, Cavicchi RV: Electrical incidents in a largehospital: a 42 month register. J Assoc Adv Med Instrum 1972, 6:222-230.

36. Leonard PF: Characteristics of electrical hazards. Anesth Analg 1972, 51:797-809.

37. Weibell FJ: Eletrical safety in the hospital - 1974. Ann Biomed Engineer 1974, 2:126-148

38. Abenstein JP: Safety While Swimming in a Sea of Energy. Mayo Clinic Proceedings 2007, 82:276-278

39. Holdstock P, Wilson N: The effect of static charge generated on hospital bedding. EOS/ESD Symposium 1996, 356-364.

40. Smallwood J: Standardisation of electrostatic test methods and electrostatic discharge prevention measures for the world market. J Electrostatics 2005, 63:501-508

41. Kathirgamanathan $\mathrm{P}$, Toohey MJ, Haase J, et al: Measurements of incendivity of electrostatic discharges from textiles used in personal protective clothing. J Electrostatics 2000, 49:51-70.

42. Eide PK: A new method for processing of continuous intracranial pressure signals. Med Eng Physics 2006, 28:579-587.

doi:10.1186/1475-925X-10-75

Cite this article as: Eide and Bakken: The baseline pressure of intracranial pressure (ICP) sensors can be altered by electrostatic discharges. BioMedical Engineering OnLine 2011 10:75.

\section{Submit your next manuscript to BioMed Central and take full advantage of:}

- Convenient online submission

- Thorough peer review

- No space constraints or color figure charges

- Immediate publication on acceptance

- Inclusion in PubMed, CAS, Scopus and Google Scholar

- Research which is freely available for redistribution 